\title{
A Comparison of Shear Bond Strength of Ceramic and Resin Denture Teeth on Different Acrylic Resin Bases
}

\author{
Massimo Corsalini ${ }^{1, *}$, Daniela Di Venere ${ }^{1}$, Francesco Pettini ${ }^{1}$, Gianluca Stefanachi ${ }^{1}$, Santo Cata- \\ pano $^{2}$, Antonio Boccaccio ${ }^{3, *}$, Luciano Lamberti $^{3}$, Carmine Pappalettere ${ }^{3}$ and Stefano Carossa ${ }^{4}$ \\ ${ }^{1}$ School of Dentistry, University of Bari, Bari, Italy \\ ${ }^{2}$ School of Dentistry, University of Ferrara, Ferrara, Italy \\ ${ }^{3}$ Department of Mechanics, Mathematics and Management (DMMM), Politecnico di Bari, Bari, Italy \\ ${ }^{4}$ School of Dentistry, University of Turin, Turin, Italy
}

\begin{abstract}
The purpose of this study is to compare the shear bond strength of different resin bases and artificial teeth made of ceramic or acrylic resin materials and whether tooth-base interface may be treated with aluminium oxide sandblasting.

Experimental measurements were carried on 80 specimens consisting of a cylinder of acrylic resin into which a single tooth is inserted. An ad hoc metallic frame was realized to measure the shear bond strength at the tooth-base interface. A complete factorial plan was designed and a three-way ANalysis Of VAriance (ANOVA) was carried out to investigate if shear bond strength is affected by the following factors: (i) tooth material (ceramic or resin); (ii) base material (self-curing or thermal-curing resin); (iii) presence or absence of aluminium oxide sandblasting treatment at the tooth-base interface. Tukey post hoc test was also conducted to evaluate any statistically significant difference between shear strength values measured for the differently prepared samples.

It was found from ANOVA that the above mentioned factors all affect shear strength. Furthermore, post hoc analysis indicated that there are statistically significant differences ( $p$-value $=0.000$ ) between measured shear strength values for: (i) teeth made of ceramic material vs. teeth made of acrylic resin material; (ii) bases made of self-curing resin vs. thermalcuring resin; (iii) specimens treated with aluminium oxide sandblasting vs. untreated specimens. Shear strength values measured for acrylic resin teeth were on average $70 \%$ higher than those measured for ceramic teeth. The shear bond strength was maximized by preparing samples with thermal-curing resin bases and resin teeth submitted to aluminium oxide sandblasting.
\end{abstract}

Keywords: Acrylic Resin Teeth, Ceramic Teeth, Resin Bases, Sandblasting, Shear Bond Strength.

\section{INTRODUCTION}

Determination of shear bond strength of denture teeth inserted into acrylic resin bases is an issue of crucial importance as most of the detachment phenomena of artificial teeth from their resin bases under functional loads occur because stresses at the tooth-base interface exceed shear bond strength. A number of studies have been reported in the literature on this important aspect [1]. Most of them analyzed the shear bond strength of artificial teeth and resin bases with similar chemical-physical properties. It was found that pretreating interfacial surfaces with dichloromethane $[2,3]$ or bonding [3,4] or using heat-cured acrylic resins [4-7],

*Address correspondence to these author at the Università degli Studi di Bari (Italy) - Dental School, Piazza Giulio Cesare, 10, I-70121 Bari, Italy; Tel: +39 0805478153; Fax: +39 0805478743;

E-mail: massimo.corsalini@uniba.it and

Department of Mechanics, Mathematics and Management (DMMM), Politecnico di Bari, Bari, Italy; Tel: +39 0805962829;

Fax: +390805962777; E-mail: a.boccaccio@ poliba.it increases the bond strength of artificial resin teeth put on acrylic resin bases [8-13]. Effects of polymerization techniques $[14,15]$, chemicals [16], surface conditioning methods and thermocycling [17-19] and surface treatments [2024] on bond strength were also investigated. A comprehensive review of the best strategies to be adopted for maximizing shear bond strength at the tooth-base interface has recently been published [25]. Correlations between the followup of removable dentures and the level of roughness of prostheses surfaces also were investigated [26, 27].

Other studies reported in the literature analyzed bond strength of ceramic denture teeth and resin bases [28-35]. Practical methods for chairside repair of debonded porcelain denture teeth were also proposed [36]. Artificial porcelain teeth do not have any chemical bond with acrylic resin, and retention between teeth and resin relies only on mechanical forces. In general, mechanical retention between artificial teeth and resin may be stronger as the configuration of interfacial surfaces becomes more complex. For example, posterior teeth may present one or more holes on their surface where soft resin penetrates during polymerization. Anterior 

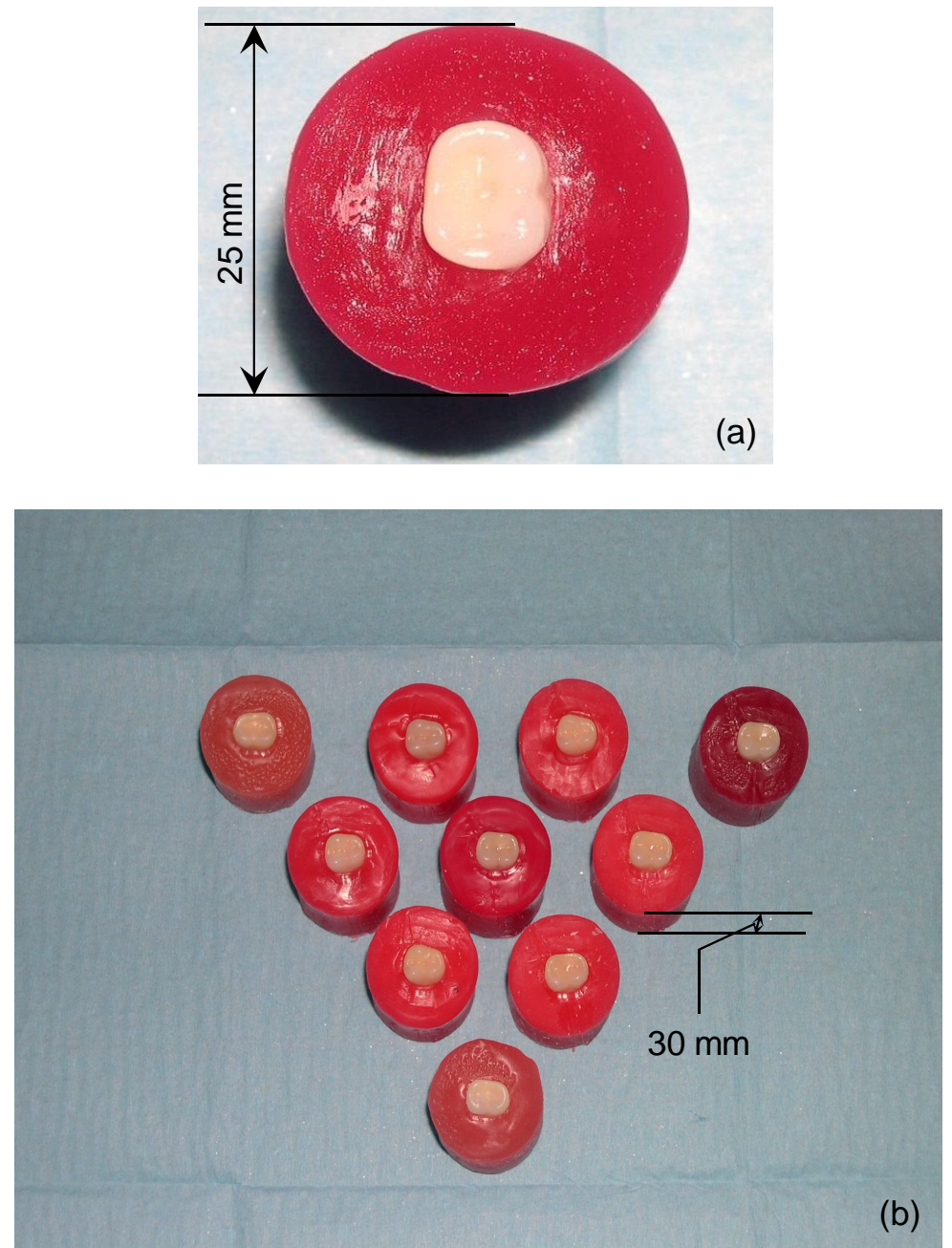

Fig. (1). (a) Details of a single specimen tested in the study; (b) samples of Group A. The main dimensions of tested samples are indicated in the figure.

teeth may instead include one or more metallic pins to be incorporated into the resin. Barpal et al. [28] found that the bond strength of ceramic anterior teeth and resin bases is comparable to that of resin anterior teeth and denture bases. Inclusion of metallic pins into ceramic teeth allowed mechanical retention to be significantly improved so that the resulting shear bond strength became comparable with that obtained via chemical bonding.

Suliman et al. [30] investigated how shear bond strength between ceramic teeth and acrylic resin cylinders depends on the treatment of interfacial surfaces: in particular, they analyzed etching with hydrofluoric acid, sandblasting with alumina sand, roughening with diamond burs, application of various bonding agents. It was found that the use of silane agents improves bond strength, however, such techniques are often too sophisticate, difficult to be implemented and yet not standardized. Lacy et al. [31] prepared specimens with ceramic teeth roughened by diamond burs and etched with hydrofluoric acid and silane. It was found that specimens roughened with diamond burs reach a high bond strength; however, the treatment may produce micro-fractures on ceramic surfaces, which can cause seepage of liquids in the interface between artificial teeth and resin base, then resulting in the detachment of the element.
The aim of the present study is to compare the shear bond strength of different resin bases and artificial teeth made of ceramic or resin materials; tooth-base interface may be treated with aluminium oxide sandblasting or not. Experimental tests were carried out on 80 specimens to investigate if and at what extent shear bond strength is affected by the following factors: (i) artificial tooth material (i.e. ceramic vs. resin); (ii) base material (i.e. self-curing resin vs. thermalcuring resin); (iii) presence or absence of aluminium oxide sandblasting treatment on the tooth surface in contact with the base. Statistical analyses with three-way ANOVA and Tukey post-hoc test were performed to interpret experimental results.

\section{MATERIALS AND METHODS}

\section{Preparation of Samples}

Resin bases were moulded into solids of cylindrical shape, $30 \mathrm{~mm}$ high and with a diameter of $25 \mathrm{~mm}$ (Fig. 1). A total of 80 samples were prepared: 40 with ceramic teeth and 40 with acrylic resin teeth. Eight groups, each of which included ten specimens, were then created and classified as follows: 
Group A with specimens realized with ceramic teeth and self-curing resin bases;

Group B with specimens realized with ceramic teeth treated with aluminium oxide sandblasting and self-curing resin bases;

Group C with specimens realized with ceramic teeth and thermal-curing resin bases treated with pressure injection moulding;

Group D with specimens realized with ceramic teeth treated with aluminium oxide sandblasting and thermalcuring resin bases treated with pressure injection moulding;

Groups E, F, G and $\mathrm{H}$ with specimens treated as for Groups A, B, C and D, respectively, but including acrylic resin teeth.

Second lower molar prosthetic teeth were utilized in the experimental tests: Condyloform CT porcelain (Candulor, Zurich, Switzerland) type for ceramic teeth and Bonartic TCR (Candulor, Zurich, Switzerland) type for resin teeth, respectively.

Specimens submitted to sandblasting treatment (Groups $\mathrm{B}, \mathrm{D}, \mathrm{F}$ and $\mathrm{H}$ ) were positioned in the sandblaster: the sandblasting operation was performed with $50 \mu \mathrm{m}$ diameter aluminium sand for 15 seconds. All samples were finished with a tungsten bur and gross and fine-grained rubber finishing tips; contact between burs and artificial teeth was carefully avoided. After finishing off processes, all samples were positioned in hermetically closed boxes filled with physiological solution. Boxes were kept away from any heat and light sources to avoid any kind of physical and mechanical stress.

The master model of the cylindrical resin bases was realized with modelling wax. A silicone duplication tray ( $\mathrm{Z} \mathrm{La}-$ bor, Zhermack SpA, Badia Polesine (Rovigo), Italy) was then realized to fabricate cylindrical specimens with the same dimensions as the master. Wax samples were randomly divided among the above described eight groups and the moulding procedure of the resin for the bases was then carried out.

The wax models utilised to fabricate the bases made of self-curing resin were put in common lab muffles after having been positioned in soft plaster for models; once the plaster hardened, the insulator Iso K (Candulor, Zurich, Switzerland) was applied and the half superior part of the muffle was positioned without the lid; at this point, the muffle was filled with additional amounts of plaster and its lid was closed. The muffle was then put for a few minutes into water at $65^{\circ} \mathrm{C}$ (i.e. the melting point of the wax utilized in this study).

The muffle was then taken out of the hot water and opened. The residual unmelted wax was removed with steam [37]. A thin layer of insulator iso $\mathrm{K}$ was applied in the muffle and the resin was then cast [38]. The resin utilized was "Aesthetic Autopolymerisat" (Candulor, Zurich, Switzerland) resin, in the amounts recommended by the manufacturer (20.5 g of polymer and $10 \mathrm{ml}$ of monomer). The resin was cast in the muffle positioned in a self-blocking stirrup. The stirrup and the muffle were put in a pressure cooker at $40^{\circ} \mathrm{C}$ temperature and 2 bar pressure to accelerate the polymerization process. After polymerization took place, samples were removed from the muffles and manually finished off by the same operator.

In the case of samples prepared with thermal-curing resin bases, the Sr-Ivocap System with the SR Ivocap Ivoclar Vivadent resin (Ivoclar-Vivadent, Schaan, Liechtenstein) was utilized following the instructions given by the manufacturer. After positioning the special space keeper into the muffles, some III class plaster was poured and samples were then put inside it removing plaster in excess. The space keeper was replaced with the thermal container of the capsule and the funnel feeding the injection channels. The surface of the lower half muffle (mould) was coated with the "Separating fluid" (Ivoclar-Vivadent, Schaan, Liechtenstein) insulator, then the upper half muffle was placed on it. The III class plaster was mixed and poured over teeth, thus avoiding the formation of bubbles. The muffle was then filled to the brim, the cover was put on and completely pressed down by hand. After the plaster had set, the muffle was plunged into hot water $\left(65^{\circ} \mathrm{C}\right)$ for more than five minutes. The muffle was opened and the remaining wax was eliminated with steam.

Two layers of insulator "Separating fluid" were applied on the surface of the plaster and the capsule of resin was prepared. To this purpose, the "Cap Vibrator" system was utilized to mix the capsule for five minutes. After this operation, the resin formed a ball. After joining the two halfmuffles together, they were inserted in the stirrup until the stop. Then, a pressure of 80 bar was applied with a hydraulic press. After removing the muffle and having connected it with the stirrup, the cartridge of resin was inserted. At this point the special injector was positioned and activated. After 5 minutes, the injector, still working, and the muffle were moved to the polymerization tub. Here, at a temperature of $100^{\circ} \mathrm{C}$, resin polymerization occurred after 35 minutes [38]. After polymerization occurred, the muffle and the injector were put in cold water for 30 minutes (the first 10 minutes were kept under pressure).

The SR Ivocap injector was therefore active during both polymerization and cooling off phases (even though for 10 minutes), because during the abovementioned phases resin is subject to retraction and other fluid resin is pushed into mould [38]. After polymerization the samples were taken out and finished off.

\section{Experimental Analyses}

The shear bond strength is a good indicator of the quality of the bond between two different materials. Stronger bond implies higher shear strength which in turns implies higher interfacial shear stress bearing capability. Different methodologies can be utilized to measure the shear bond strength, that include, for example, micro-tensile [19, 39], micro pushout [40] and pull-out tests [41].

In this study, an ad hoc metallic frame was designed and realized (Fig. 2) to measure shear strength. Tests were performed with a Universal Testing Machine Instron 4485 equipped with a $100 \mathrm{kN}$ load cell. The frame includes two 

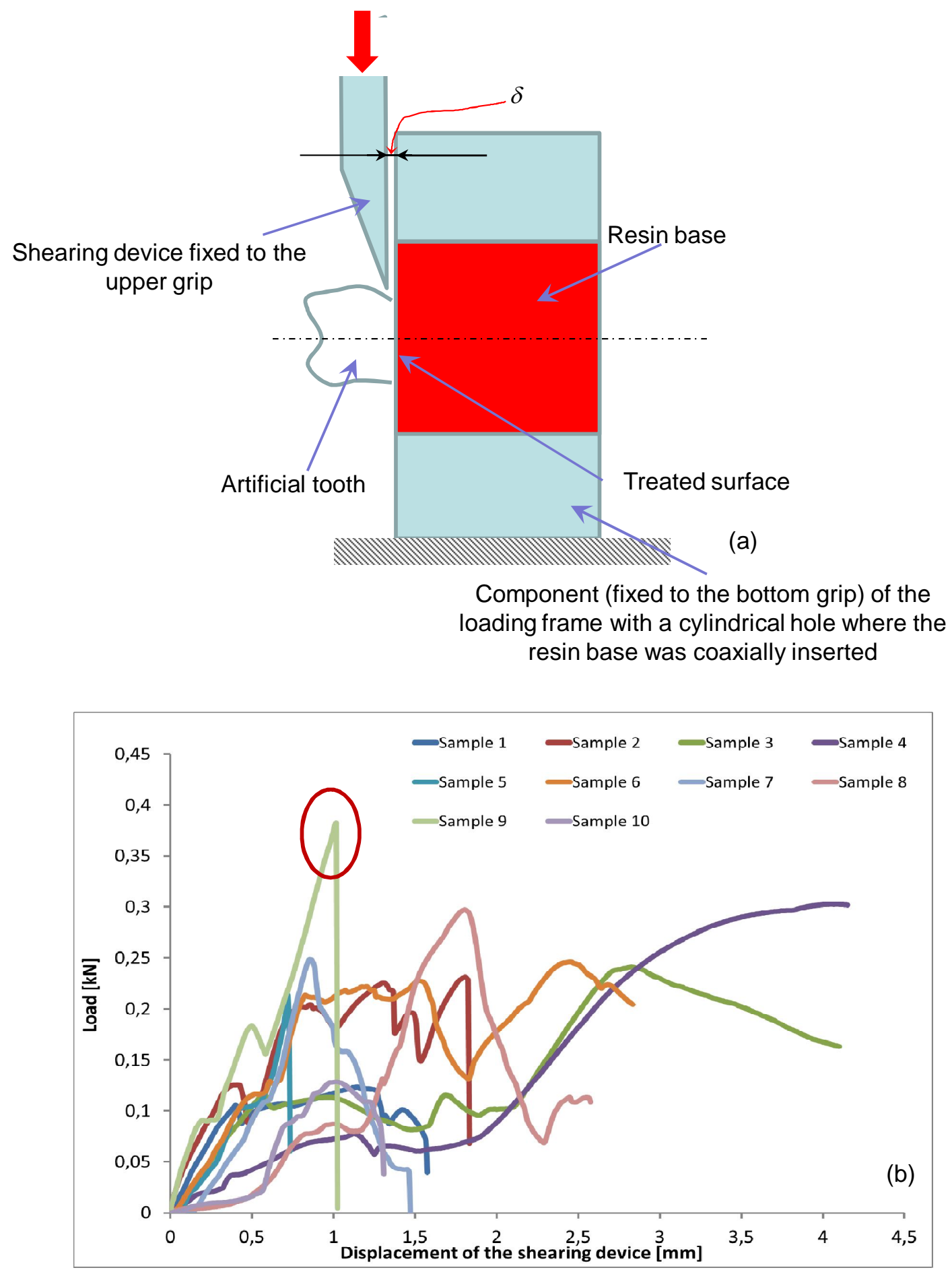

Fig. (2). Schematic (a) of the loading frame utilized to determine the shear bond strength at the tooth-base interface; (b) Force-displacement curves recorded for Group A samples.

components fixed to the upper and bottom grips, respectively. The latter serves to keep in position the cylindrical base with the artificial tooth attached to it while the former has a shearing device that during the test moves at a constant speed towards the inferior grip (Fig. 2a). In order to make parasitic bending effects negligible, special care was taken in minimizing the distance $\delta$ (Fig. 2a) between the shearing device and the flat surface of the resin base. The crosshead speed was set equal to $5 \mathrm{~mm} / \mathrm{min}$ and force-displacement curves of the shearing device (Fig. 2b) were recorded at a frequency of $10 \mathrm{~Hz}$. Each test continued until detachment of the artificial tooth occurred. The corresponding shear strength $(S S)$ was computed as the ratio between the value of the load $L_{f}$ acting on the sample (that produces the slippage of the tooth on the resin base) immediately before the failure (see, for example, Fig. (2b) where $L_{f}$ for the sample 9 is circled in red) and the area $A_{t}$ of the transverse section of the artificial tooth: 


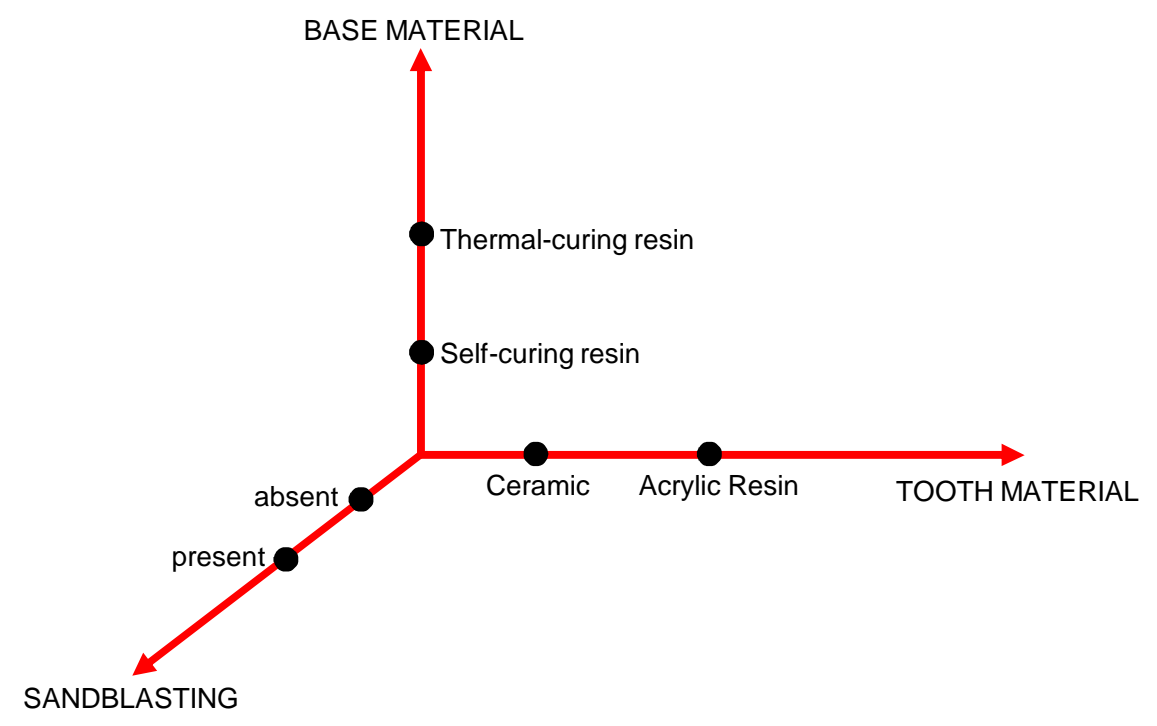

Fig. (3). Complete factorial plan designed to identify factors that have a statistically significant influence on the shear bond strength at the tooth-base interface.

$$
S S=\frac{L_{f}}{A_{t}}
$$

By expressing the load in $\mathrm{N}$ and the area of the tooth section in $\mathrm{mm}^{2}$, the shear bond strength can be expressed in MPa.

Experimental tests were carried out in an acclimatized environment at constant temperature of $23^{\circ} \mathrm{C}$ and $50 \%$ humidity. Specimens were all tested in the same day.

\section{Identification of the Factors Affecting Shear Bond Strength: Statistical Analysis}

The shear tests conducted in the experimental campaign were aimed at assessing, by means of three-way ANalysis Of VAriance (ANOVA), the factors that may affect shear bond strength of artificial teeth on resin bases. A complete factorial plan was designed with respect to the following three factors: (i) artificial tooth material (i.e. ceramic vs. resin); (ii) resin base material (i.e. self-curing vs. thermally cured resins); (iii) presence or absence of aluminium oxide sandblasting treatment at the tooth-base interface. The null hypothesis $\mathrm{H}_{\mathrm{o}}$ was that factors (i), (ii) and (iii) do not affect shear strength. $H_{o}$ was assumed to hold true for p-values $\geq 0.05$ (i.e. 95\% confidence interval). Two levels were set for each factor. In particular, for factor (i): artificial teeth made of resin or ceramic; for factor (ii): bases made of self-curing resin or thermal-curing resin treated with pressure injection moulding; for factor (iii): with or without aluminium oxide sandblasting treatment. The factors forming the factorial plan and their corresponding levels are summarized in Fig. (3). A total of 80 experimental tests, 2 (base material) $\times 2$ (tooth material) $\times 2$ (with or without sandblasting $) \times 10$ (repetitions), were conducted as each experiment corresponding to a particular combination of factors and levels was repeated ten times.
The number of samples (i.e. sample size $=10$ ) tested for each group was computed with the "Sample Size for Estimation" module available in the Minitab ${ }^{\circledR}$ Version 17 software utilized in this study for statistical analysis. By choosing as parameter of interest the mean that was hypothesized to follow a normal distribution and by assuming a $5 \mathrm{MPa}$ standard deviation of experimental data, a confidence level of $95 \%$ and a two-sided confidence interval, the sample size was computed as 10 . This value is comparable (or even larger) with those adopted by other authors in similar studies reported in the literature $[30,31,33]$.

A non-parametric test (analysis of variance on ranks with Tukey post-hoc test) was finally performed in order to assess the level of correlation between shear bond strength values measured: (a) with or without sandblasting; (b) with bases made of self-curing or thermal-curing resins; (c) with artificial teeth made of ceramic or resin.

\section{RESULTS AND DISCUSSION}

In this study, a loading frame was designed and realized to measure the shear bond strength between artificial teeth and acrylic resin bases. The relative performance of eight dental repairs including various combinations of tooth and base materials and tooth-base interface treatments was analyzed on a statistical basis. The three-way ANOVA carried out on the data gathered in the experimental tests reveals that tooth material, resin base type and the presence/absence of sandblasting treatment all affect significantly the shear bond strength at the tooth-base interface. This is confirmed by Table 1: the p-value is in fact 0.000 for all of the three above mentioned factors. The measured shear strength tests is higher for resin teeth (with respect to ceramic teeth), for thermal-curing resin bases (with respect to self-curing resin bases) and in presence of aluminium oxide sandblasting treatment (see Fig. 4), respectively. For each groups of samples, the median value, first and third quartiles, minimum 
Table 1. Three-way ANOVA of shear bond strength experimental data.

\begin{tabular}{|c|c|c|c|c|c|}
\hline Source & $d f$ & SS & MS & F-value & P-value \\
\hline Tooth material & 1 & 662.6 & 662.58 & 19.64 & 0.000 \\
\hline Base material & 1 & 577.6 & 577.61 & 17.12 & 0.000 \\
\hline Sandblasting & 1 & 835.1 & 835.09 & 24.76 & 0.000 \\
\hline Error & 76 & 2563.5 & 33.73 & & \\
\hline Lack-of-fit & 4 & 247.5 & 61.88 & 1.92 & 0.116 \\
\hline Pure error & 72 & 2316.0 & 32.17 & & \\
\hline Total & 79 & 4638.8 & & & \\
\hline
\end{tabular}

$\mathrm{SS}=$ Sum of squares; MS= Mean of squares
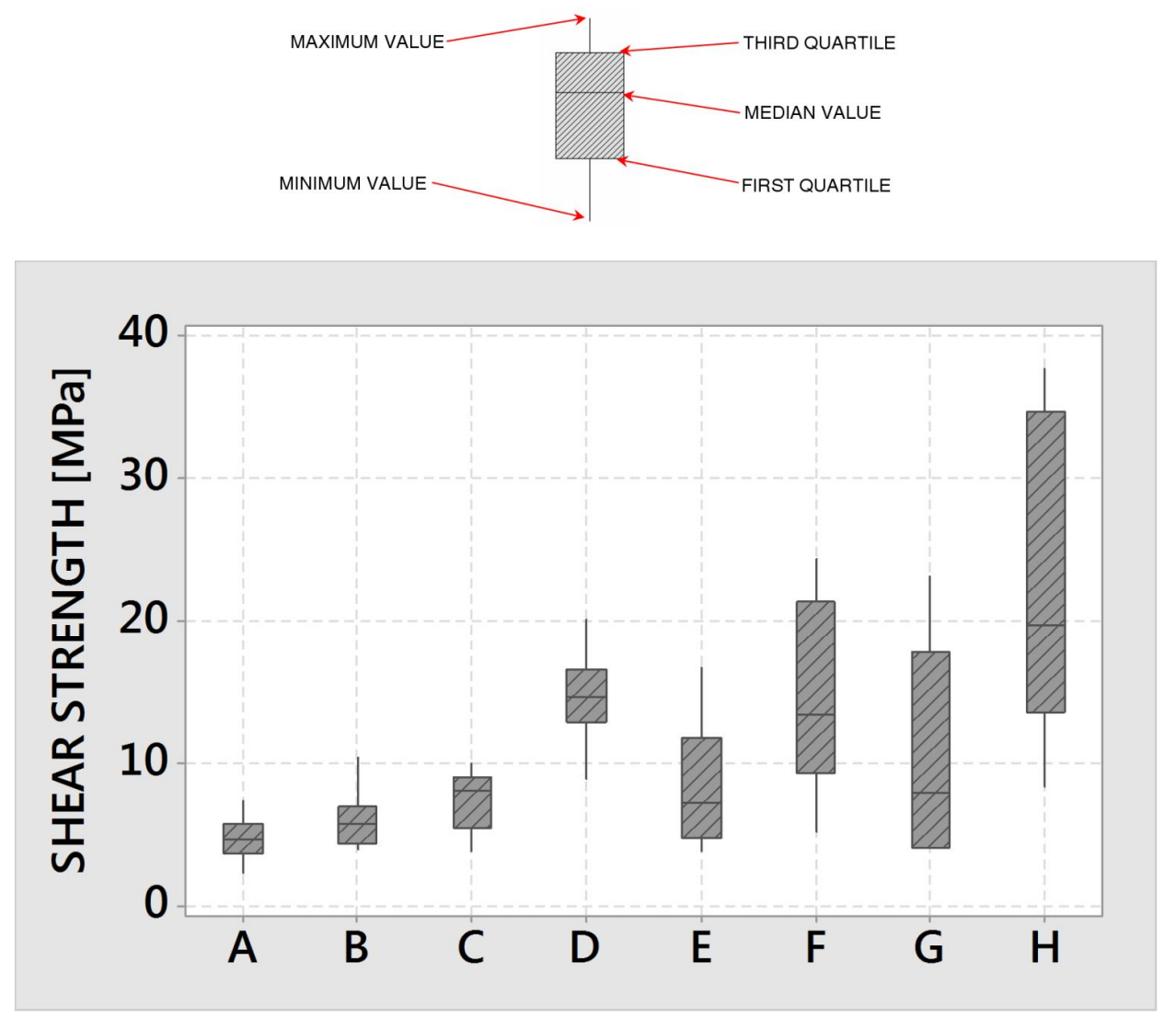

Fig. (4). Boxplot of shear bond strength values determined for the different groups of tested samples and detail illustrating median value, first and third quartiles, minimum and maximum values measured in the experiments.

Table 2. Average shear bond strength values and corresponding standard deviations measured for each of the investigated groups.

\begin{tabular}{|c|c|c|c|c|c|c|c|c|}
\hline & Group A & Group B & Group C & Group D & Group E & Group F & Group G & Group H \\
\hline \hline Average [MPa] & 4.624 & 5.976 & 7.399 & 14.682 & 8.393 & 14.452 & 10.852 & 22.007 \\
\hline Standard Deviation [MPa] & 1.492 & 1.970 & 2.019 & 3.277 & 4.200 & 6.724 & 7.538 & 10.804 \\
\hline
\end{tabular}

and maximum values of measured shear strength are indicated according to the nomenclature shown in the upper part of the figure.
Table 2 lists the average strength values measured in the experimental tests and the corresponding standard deviations (computed over the 10 repetitions of each experiment) for 


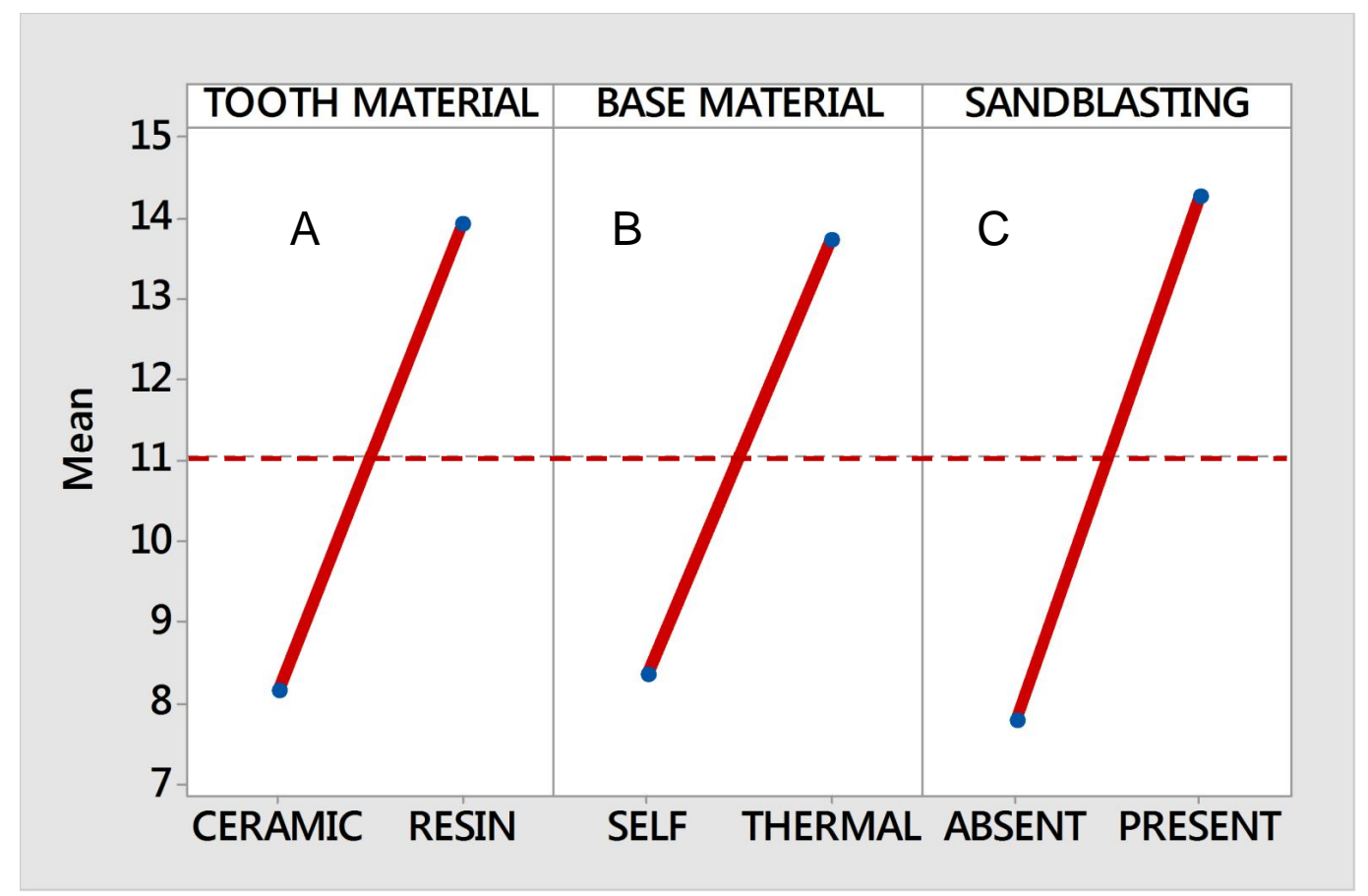

Fig. (5). Main effects plot of shear bond strength values averaged over experiments carried out with respect to different toot/resin base materials and sample preparation protocols.

each group of specimens. The average values of shear strength determined for each factor are shown in the main effects plot of Fig. (5) where the dashed horizontal line corresponds to the average strength obtained for the whole set of 80 specimens. For example, the value of shear strength reported in diagram A for the "ceramic" teeth is the average strength value measured for all samples including artificial teeth made of ceramic. Similarly, the strength value reported in diagram $\mathrm{C}$ for the "sandblasting treatment present" was averaged for all samples including a tooth-base interface treated with aluminum oxide sandblasting. Similar interpretations can be made for all data shown in Fig. (5).

The Tukey post-hoc test revealed that statistically significant differences exist in shear strength values measured in all cases: (i) teeth made of ceramic or resin; (ii) bases made of self-curing or thermal-curing resin; (iii) teeth treated with aluminium oxide sandblasting or not (Fig. 6). The resulting p-value always was 0.000 .

As mentioned above, bond shear strength significantly depends on the chosen combination of tooth material, base material and sandblasting treatment. In particular, the average shear strength of the 40 samples submitted to sandblasting was $82.7 \%$ higher than the average strength measured for the untreated specimens (i.e. 14.279 vs. $7.817 \mathrm{MPa}$ ). This is because sandblasting allows the surface of the tooth in contact with the resin base to be enlarged thus improving the adhesion between the two surfaces. A further proof of the efficiency of the sandblasting treatment is that the Tukey post-hoc test (Fig. 6) reveals that statistically significant differences exist between treated and untreated specimens.

The fabrication technique utilized for the resin bases affects shear strength less significantly than the sandblasting treatment. In particular, in the case of thermal-curing resins treated with pressure injection moulding, the average bond strength became $64.3 \%$ higher than its counterpart measured for specimens including self-curing resin bases (i.e. 13.735 vs. 8.361 MPa). The observed differences again were statistically very significant (see the results of the Tukey post-hoc shown in Fig. 6). Such a behaviour occurs because the thermal-curing resin treated with pressure injection moulding can penetrate more deeply in the tooth micro-cavities thus allowing the resin to adapt itself to the dental surface [42]. Tooth retention may further be improved with the sandblasting pre-treatment which increases the number of microcavities that can be occupied by the resin. Remarkably, the thermal-curing resin has a very low polymerization retraction coefficient: this does not interfere with the different thermal expansion of ceramic teeth thus producing no residual stresses at the tooth-base interface.

The selection of tooth material (ceramic vs. acrylic resin) affects bond shear strength more significantly than the fabrication process chosen for the resin bases but less significantly than the use of sandblasting treatment. In particular, the average value of $S S$ measured for teeth made of acrylic resin was $70.5 \%$ higher than that found for ceramic teeth (i.e. 13.926 vs. $8.170 \mathrm{MPa}$ ). Experimental data were found to be statistically different by performing the Tukey post-hoc test (see Fig. 6). The lower performance of specimens including ceramic teeth can be explained with the lack of chemical bonding between ceramic and resin components [43]. Different studies [30, 31] investigated on how to create a hybrid transition layer between ceramic and resin thus allowing a chemical bond between the two materials to be generated; however, these methods entail sophisticate procedures and the utilization of materials like primer, bonding and silane. Since these materials were not originally conceived for dental applications but only for orthodontic scopes, the protocols 


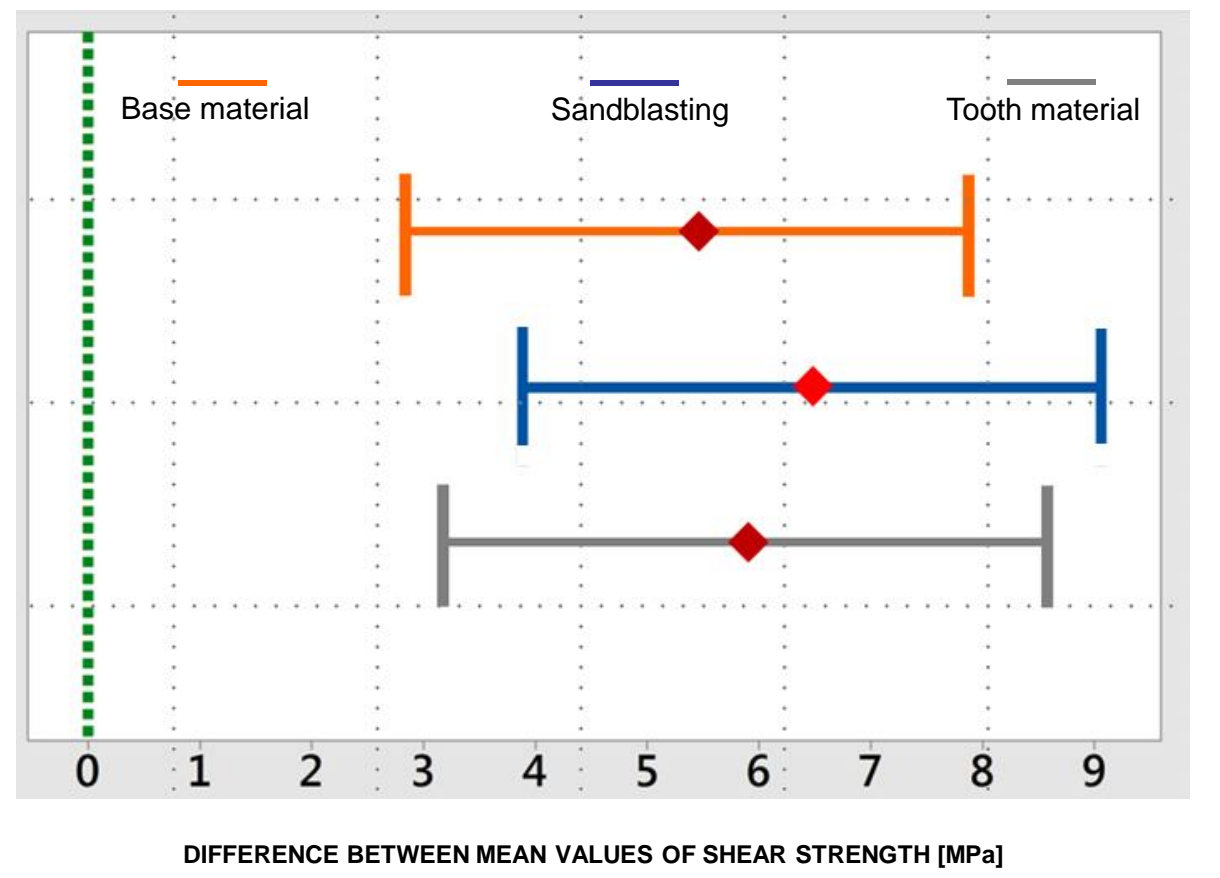

Fig. (6). Interval plots for differences of means of experimental data gathered for samples including acrylic resin or ceramic teeth, thermalcuring or self-curing base resin, treated or not with aluminium oxide sandblasting. If the difference interval does not contain zero, the corresponding means are significantly different.

for their use are not standardized and remain empirical and untested by manufacturers.

The factorial plan set in this study is rather comprehensive although the present investigation has some limitations. In particular, values of shear bond strength were measured in-vitro hence not accounting for the effects of saliva, acids, residues of food and other substances present in the oral cavity. Furthermore, mastication is a dynamic process and the adhesion behaviour at the tooth-base interface should be characterized in terms of fatigue. Bond strength values measured in vitro in this study actually are an over-estimate of the real values that would be measured in vivo. However, in spite of the care taken in minimizing the distance $\delta$ between the shearing device and the flat surface of the resin base (Fig. 2a), the parasitic effects of the bending moment did not remain null. This somehow made the in vitro results closer to the real scenario as parasitic bending reduces the shear strength. All of the above mentioned issues will be the subject of future investigations. However, the very large and statistically significant differences observed in the experimental data gathered for the tested combinations of tooth/resin base materials and specimen preparation suggest that the relative behaviour of the eight groups of samples considered in this study should not change significantly if tests were conducted in vivo.

Another concern may be on the crosshead speed adopted in the experiments. According to literature [30,31], a rather low value of $5 \mathrm{~mm} / \mathrm{min}$ was chosen in order to minimize the possible visco-elastic effects that could have been involved in the mechanical response of tested samples. Ideally, the crosshead speed should tend towards zero so that specimens can be tested in "static" conditions. However, the values of shear strength measured experimentally were also consistent with data reported in the literature for lower crosshead speeds [33].

As far as it concerns sample preparation, sandblasting was performed with $50 \mu \mathrm{m}$ aluminium sand for 15 seconds. Different protocols for the sandblasting treatment are documented in literature [44]. It would be interesting to study the sensitivity of the shear bond strength to the different process parameters involved in the sandblasting treatment (e.g. exposure time, dimension of the sand etc) for the specific materials analyzed in this study. For example, recently published results [45] show that no statistically significant differences exist between values of $S S$ measured on samples (yet prepared with materials different from those utilized in this study) submitted to sandblasting treatment for different time intervals. Another limitation is that the effect of the artificial ageing treatment was not analysed. This aspect will be the object of future investigations.

Amongst the different methodologies utilized to determine the bond strength between two materials, the microtensile test [19] has a prominent place. Interestingly, the shear bond strength values measured in this study were of the same order of magnitude of tensile bond strength values reported in [19]. The implementation of finite element models simulating the boundary and loading conditions acting in each specific test may help to better understand how the mechanical response of the tested samples changes under different testing conditions. Similar approaches were successfully adopted in previous studies [40, 46-49].

\section{CONCLUSION}

1. The shear bond strength at the tooth-base interface significantly depends on the following factors: 
(i) tooth material; (ii) base material; (iii) sandblasting treatment.

2. Statistically significant differences $(p$-value $=0.000)$ exist between shear bond strength values measured for teeth made of different materials (ceramic vs. acrylic resin), bases made of self-curing or thermal-curing resin, teeth treated with aluminium oxide sandblasting or not.

3. The highest values of shear bond strength were found in the case of teeth made of acrylic resin, bases made of thermal-curing resin (treated with pressure injection moulding) and teeth submitted to aluminium sandblasting.

4. Sandblasting allows shear strength to be increased on average by $82.7 \%$ with respect to untreated specimens.

5. Utilizing acrylic resin teeth allows shear strength to be increased on average by $70.4 \%$ with respect to specimens including ceramic teeth.

6. Utilizing thermal-curing resins in conjunction with pressure injection moulding allows shear strength to be increased on average by $64.3 \%$ with respect to specimens including self-curing resin bases.

7. Within the limitations entailed by this investigation, the comprehensive factorial plan developed in the research has provided further evidence on how shear bond strength of prosthetic teeth may vary during normal working conditions of the prosthesis.

\section{CONFLICT OF INTEREST}

The authors confirm that this article content has no conflicts of interest.

\section{ACKNOWLEDGEMENTS}

Declared none.

\section{REFERENCES}

[1] Cunningham JL. Bond strength of denture teeth to acrylic bases (review). J Dent 1993; 21: 274-80.

[2] Chai J, Takahashi Y, Takahashi T, Habu T. Bonding durability of conventional resinous denture teeth and highly crosslinked denture teeth to a pour-type denture base resin. Int J Prosthodont 2000; 13: 112-6.

[3] Takahashi Y, Chai J, Takahashi T, Habu T. Bond strength of denture teeth to denture base resins. Int J Prosthodont 2000; 13: 59-65.

[4] Cunningham JL, Benington IC. Bond strength variation of synthetic resin teeth in dentures. Int J Prosthodont 1995; 8: 69-72.

[5] Cunningham JL, Benington IC. An investigation of the variables which may affect the bond between plastic teeth and denture base resin. J Dent 1999; 27: 129-35.

[6] Cunningham JL. Shear bond strength of resin teeth to heat-cured and light-cured denture base resin. J Oral Rehabil 2000; 27: 312-6.

[7] Clancy JMS, Boyer DB. Comparative bond strengths of lightcured, heat-cured, and autopolymerizing denture resins to denture teeth. J Prosthet Dent 1989; 61: 457-62.

[8] Huggett R, John G, Jagger RG, Bates JF. Strength of the acrylic denture base tooth bond. Br Dent J 1982; 153: 187-90.

[9] Spratley MH. An investigation of adhesion of acrylic resin teeth to dentures. J Prosthet Dent 1987; 58: 389-92.
[10] Kawara M, Carter JM, Ogle RE, Johnson RR. Bonding of plastic teeth to denture base resins. J Prosthet Dent 1991; 66: 566-71.

[11] Vallittu PK. Bonding of resin teeth to the polymethyl methacrilate denture base material. Acta Od Scand 1995; 53: 99-104.

[12] Buyukyilmaz S, Ruyter IE. The effects of polymerization temperature on the acrylic resin denture base-tooth bond. Int J Prosthodont 1997; 10: 49-54.

[13] Vallittu PK, Ruyter IE. The swelling phenomenon of acrilic resin polymer teeth at the interface with denture base polymers. J Prosthet Dent 1997; 78: 194-9.

[14] Fletcher-Stark ML, Chung KH, Rubenstein JE, Raigrodski AJ, Mancl LA. Shear bond strength of denture teeth to heat- and lightpolymerized denture base resin. J Prosthodont 2011; 20:52-9.

[15] Bahrani F, Khaledi AA. Effect of surface treatments on shear bond strength of denture teeth to denture base resins. Dent Res J (Isfahan) $2014 ; 11: 114-8$.

[16] Krishna VP, Premalatha A, Babu PJ, Raju DS, Kumar MP, Rao DB. Effect of various chemicals on the bond strength of acrylic tooth and denture base -An Invitro comparative study. J Int Oral Health 2014; 6: 100-5.

[17] Marra J, de Souza RF, Barbosa DB, Pero AC, Compagnoni MA. Evaluation of the bond strength of denture base resins to acrylic resin teeth: effect of thermocycling. J Prosthodont 2009; 18: 43843.

[18] Madhav GV, Raj S, Yadav N, Mudgal I, Mehta N, Tatwadiya R Shear bond strength of acrylic teeth to acrylic denture base after different surface conditioning methods. J Contemp Dent Pract 2013; 14: 892-7.

[19] Saavedra G, Valandro LF, Leite FP, et al. Bond strength of acrylic teeth to denture base resin after various surface conditioning methods before and after thermocycling. Int J Prosthodont 2007; 20: 199-201.

[20] Bragaglia LE, Prates LH, Calvo MC. The role of surface treatments on the bond between acrylic denture base and teeth. Braz Dent J 2009; 20: 156-61.

[21] Kurt M, Saraç YŞ, Ural C, Saraç D. Effect of pre-processing methods on bond strength between acrylic resin teeth and acrylic denture base resin. Gerodontology 2012; 29: e357-62.

[22] Akin H, Tugut F, Guney U, Akar T. Shear bond strength of denture teeth to two chemically different denture base resins after various surface treatments. J Prosthodont 2014; 23: 152-6.

[23] Chatterjee N, Gupta TK, Banerjee A. A study on effect of surface treatments on the shear bond strength between composite resin and acrylic resin denture teeth. J Ind Prosthodont Soc 2011; 11: 20-5.

[24] Consani RL, Naoe HT, Mesquita MF, Sinhoreti MA, Mendes WB. Effect of ridge-lap surface treatments on the bond of resin teeth to denture base. J Adhes Dent 2011; 13: 287-93.

[25] Radford DR, Juszczyk AS, Clark RK. The bond between acrylic resin denture teeth and the denture base: recommendations for bes practice. Br Dent J 2014; 216: 165-7.

[26] Corsalini M, Carella M, Boccaccio A, et al. An alternative approach to the polishing technique for acrylic resin surfaces. Int J Prosthodont 2008; 21: 409-12.

[27] Corsalini M, Boccaccio A, Lamberti L, Pappalettere C, Catapano S, Carossa S. Analysis of the performance of a standardized method for the polishing of methacrylic resins. Open Dent J 2009; 3: 23340.

[28] Barpal D, Curtis DA, Finzen F, Perry J, Gansky SA. Failure load of acrylic resin denture teeth bonded to high impact acrylic resins. J Prosthet Dent 1998; 80: 666-71.

[29] Moffa JP, Jenkins WA, Weaver RG. Silane bonding of porcelain denture teeth to acrylic resin denture bases. J Prosthet Dent 1975; 33: 620-7.

[30] Suliman AHA, Swift EJ, Perdigao J. Effects of surface treatment and bonding agents on bond strength of composite resin to porcelain. J Prosthet Dent 1993; 70: 118-20.

[31] Lacy AM, La Luz J, Watanabe LG, Dellinges M. Effect of porcelain surface treatment on the bond to composite. J Prosthet Dent 1998; 60: 288-91.

[32] El-Sheikh MM, Powers JM. Tensile bond strength of porcelain teeth to denture resin before and after aging. Int $\mathbf{J}$ Prosthodont 1998; 11: 16-20.

[33] Marchack WB, Yu Z, Zhao XY, White SN. Adhesion of denture tooth porcelain to heat-polymerized denture resin. J Prosthet Dent 1995; 74: 242-9. 
[34] Sumithra N, Waknine S, Schulman A. Bond strength of etched porcelain denture teeth to PMMA. Quint Int 1986; 17: 745-8.

[35] El-Sheikh M, Powers J. Tensile bond strength of four denture resins to porcelain teeth with different surface treatment. J Adv Prosthodont 2013; 5: 423-7.

[36] Ozcan M. A practical method for chairside repair of debonded porcelain denture teeth. J Prosthodont 2006; 15: 47-50.

[37] Cunningham JL, Benington IC. A survey of the pre-bonding preparation of denture teeth and the efficiency of dewaxing methods. J Dent 1997; 25: 125-8.

[38] Salim S, Sadamori S, Hamada T. The dimensional accuracy of rectangular acrylic resin specimens cured by three denture base processing methods. J Prosthet Dent 1992; 67: 879-81.

[39] Goracci C, Tavares AU, Fabianelli A, et al. The adhesion between fiber posts and root canal walls: comparison between microtensile and push-out bond strength measurements. Eur J Oral Sci 2004; 112: 353-61.

[40] Grassi FR, Pappalettere C, Di Comite M, et al. Effect of different irrigating solutions and endodontic sealers on bond strength of the dentin-post interface with and without defects. Int J Med Sci 2012; 9: 642-54.

[41] Varela GS, Rabade BL, Lombardero RP, Sixto LJ, Bahillo GJ, Park AS. In vitro study of endodontic post cementation protocols that use resin cements. J Prosthet Dent 2003; 89: 146-53.
[42] Monfrin BS, Notaro V, Gassino G, Perotti R, Bassi F. Dimensional contour stability of acrylic resin bases for complete dentures before and after water sorption. Int J Prosthodont 2005; 18: 480-2.

[43] Zappini G, Kammann A, Wachter W. Comparison of fracture tests of denture base materials. J Prosthet Dent 2003; 90:578-85.

[44] Chung KH, Chung CY, Chung CY, Chan DC. Effect of preprocessing surface treatments of acrylic teeth on bonding to the denture base. J Oral Rehabil 2008; 35: 268-75.

[45] Salehi AE, Heshmat H, Salehi ME, Kharazifard MJ. In vitro evaluation of the effect of different sandblasting times on the bond strength of feldspathic porcelain to composite resin. J Isl Dent Assoc IRAN (JIDA) 2013; 25: 12-4

[46] Boccaccio A, Frassanito MC, Lamberti L, et al. Nanoscale characterization of the biomechanical hardening of bovine zona pellucida. J R Soc Interface 2012; 9: 2871-82.

[47] Boccaccio A, Casavola C, Lamberti L, Pappalettere C. Structural response of polyethylene foam-based sandwich panels subjected to edgewise compression. Materials 2013; 6: 4545-64.

[48] Gracco A, Cirignaco A, Cozzani M, Boccaccio A, Pappalettere C, Vitale G. Numerical/experimental analysis of stress field around mini-screws for the orthodontic anchorage. Eur J Orthod 2009; 31: 12-20.

[49] Boccaccio A, Lamberti L, Pappalettere C, Quagliarella L. Accuracy of finite element predictions on bone/implant interface contact pressures for models reconstructed from CT scans. J Mech Med Biol 2008; 8: 161-82.

Received: September 19, 2014

Revised: November 07, 2014

Accepted: November 17, 2014

(c) Corsalini et al.; Licensee Bentham Open.

This is an open access article licensed under the terms of the Creative Commons Attribution Non-Commercial License (http://creativecommons.org/licenses/by-nc/3.0/) which permits unrestricted, non-commercial use, distribution and reproduction in any medium, provided the work is properly cited. 